\title{
AN EXAMPLE OF ANOMALOUS SINGULAR HOMOLOGY
}

\author{
M. G. BARRATT AND JOHN MILNOR ${ }^{1}$
}

Introduction. The Cech homology groups of an $r$-dimensional space are known to vanish in dimensions greater than $r$. We will show that the corresponding assertion for the singular homology groups is false, even on the category of locally $(r-1)$-connected compacta. An $r$ dimensional compactum $X$, locally contractible save at one point, is given such that the singular homology groups $H_{q}(X ; Q)$ with rational coefficients are nontrivial for infinitely many values of $q$. This settles a question raised by Eilenberg and Steenrod [1, Problem 22].

Let $X$ denote the union of a countable number of $r$-spheres $(r>1)$ with a single point in common and a metric topology such that the diameter of the spheres tends to zero with increasing index. This example was suggested by Steenrod. Our result is

THEOREM 1. The rational singular homology groups $H_{q}(X ; Q)$ with $q \equiv 1 \bmod (r-1), q>1$, are not zero. ${ }^{2}$ In fact these groups are not even countable.

The proof, which applies to bouquets of a more general nature, is based on the composition

$$
\omega_{Q}: \pi_{q}(X) \rightarrow H_{q}(X ; Q)
$$

of the Hurewicz homomorphism $\omega: \pi_{q}(X) \rightarrow H_{q}(X)$ and the coefficient homomorphism induced by the inclusion $Z \rightarrow Q$ of the integers in the rationals. It is shown that $\omega_{Q}$ is not trivial.

1. Two observations. If $X$ is any simply-connected space, the kernel of the Hurewicz homomorphism $\omega: \pi_{q}(X) \rightarrow H_{q}(X)$, can be characterized as follows. Let $\alpha$ be an element of $\pi_{q}(X)$, where $q>1$.

LEMMA 1. $\omega(\alpha)=0$ if and only if there exists a finite polyhedron $K$ of dimension $<q$, and a map $f: K \rightarrow X$ such that $\alpha \in f_{*} \pi_{q}(K)$.

Proof. In the exact sequence

$$
\cdots \rightarrow \Gamma_{q}(S X) \rightarrow \pi_{q}(S X) \stackrel{\omega}{\rightarrow} H_{q}(S X) \rightarrow \cdots
$$

Received by the editors February 20, 1961.

1 The second author held an Alfred P. Sloan Fellowship.

2 It soems plausible to conjecture that $H_{q}(X)=0$ for $q \neq 1 \bmod (r-1), q>0$. It is not difficult to prove this for $r<q<2 r-1$. 
(see [3]; here $S X$ denotes the singular complex of $X$ as there defined), the image of $\Gamma_{q}$ in $\pi_{q}$ is the image of $\pi_{q}\left((S X)^{q-1}\right)$ in $\pi_{q}(S X)$ under the inclusion of the $(q-1)$-skeleton $(S X)^{q-1}$ of $S X$ in $S X$. Since the image of a map $S^{q} \rightarrow(S X)^{q-1}$ is compact, it lies in a finite subcomplex $K \subset(S X)^{q-1}$. Therefore the lemma is true for $S X$. It follows for $X$ also, since the natural map $S X \rightarrow X$ induces isomorphisms of the homotopy and singular homology groups.

Let $k: S^{q} \rightarrow S^{m} \bigvee S^{n}(q=m+n-1)$ represent the Whitehead product $\left[\iota_{m}, \iota_{n}\right]$ of the generators of $\pi_{m}\left(S^{m}\right), \pi_{n}\left(S^{n}\right)$; let $a_{m}, b_{n}$ similarly generate $H^{m}\left(S^{m} ; \Lambda\right), H^{n}\left(S^{n} ; \Lambda\right)$, regarded as subgroups of the corresponding groups of $S^{m} \bigvee S^{n}$, where $\Lambda$ is a ring. Then $a_{m} \cup b_{n}=0$, and the functional cup-product [2]

$$
c_{q}=a_{m} \bigcup_{k} b_{n} \in H^{q}\left(S^{q} ; \Lambda\right)
$$

is defined and generates this cohomology group.

More generally, if $\alpha \in \pi_{m}(X), \beta \in \pi_{n}(X)$, the Whitehead product $[\alpha, \beta]$ can be represented by a map $f=g \circ k$ :

$$
S^{q} \stackrel{k}{\rightarrow} S^{m} \vee S^{n} \stackrel{g}{\rightarrow} X
$$

Given cohomology classes $u \in H^{m}(X ; \Lambda), v \in H^{n}(X ; \Lambda)$ with $u \cup v=0$, the functional cup-product

$$
u \bigcup_{f} v \in H^{q}\left(S^{q} ; \Lambda\right)
$$

is defined and equal to $g^{*} u \cup_{k} g^{*} v$. This is because $f^{*}: H^{q}(X ; \Lambda)$ $\rightarrow H^{q}\left(S^{q} ; \Lambda\right)$ is trivial, since $k^{*}$ is necessarily trivial on $H^{q}\left(S^{m} \vee S^{n} ; \Lambda\right)$.

LEMmA 2. $u \cup_{f} v=\lambda c_{q}$, where $c_{q}$ generates $H^{q}\left(S^{q} ; \Lambda\right)$ and

$$
\lambda=\left\{\begin{array}{l}
\langle u, \omega \alpha\rangle\langle v, \omega \beta\rangle \quad \text { if } n \neq m, \\
\langle u, \omega \alpha\rangle\langle v, \omega \beta\rangle+(-1)^{n}\langle u, \omega \beta\rangle\langle v, \omega \alpha\rangle \text { if } n=m .
\end{array}\right.
$$

As usual, $\langle u, z\rangle$ denotes $h(u)(z)$, where $h: H^{p}(X ; \Lambda) \rightarrow \operatorname{Hom}\left(H_{p}(X) ; \Lambda\right)$ is the projection. The proof is easy.

2. Bouquets with the strong topology. Let $\left(A_{\xi}\right)(\xi \in \Xi)$ be a family of spaces, each with a specified base point $*$, indexed to an infinite set $\Xi$. The bouquet

$$
Y=\bigvee_{\xi \in Z} A_{\xi}
$$

will mean the subset of the cartesian product $\prod_{\xi \in \Xi} A_{\xi}$ which consists of all points $\left(a_{\xi}\right)$ such that $a_{\xi} \neq *$ for at most one value of $\xi$. We identify each $A_{\xi}$ with the corresponding subset of $Y$. The result- 
ing topology is stronger than the usual weak topology; this space has the properties:

(1) There are embeddings $A_{\eta} \rightarrow Y$ and projections $Y \rightarrow A_{\zeta}$, such that the compositions $A_{\eta} \rightarrow Y \rightarrow A_{\zeta}$ are the identity maps if $\eta=\zeta$ and trivial otherwise.

(2) Any neighbourhood of the common point * contains all but a finite number of the $A_{\xi}$.

From (1) it follows that we may regard $\pi_{q}\left(A_{\xi}, *\right), H_{q}\left(A_{\xi}\right), H^{q}\left(A_{\xi} ; Q\right)$ as embedded as direct summands in $\pi_{q}(Y, *), H_{q}(Y), H^{q}(Y ; Q)$. From (2) it follows that if $J=\{1,2,3, \ldots\} \subset \Xi$ is a countable subset, and if elements $\alpha_{n} \in \pi_{q}\left(A_{n}, *\right), n \in J$, are given, we may form the sum

$$
\alpha=\sum_{n \in J} \alpha_{n}
$$

(the order being unimportant if $q>1$ ). This can be defined by a representative map $f:\left(I^{q}, \dot{I}^{q}\right) \rightarrow(Y, *)$ given by

$$
f\left(t_{1}, \cdots, t_{q}\right)=f_{n}\left(n(n+1)\left(t_{1}-1\right)+n+1, t_{2}, \cdots, t_{q}\right)
$$

for $1-1 / n \leqq t_{1} \leqq 1-1 /(n+1), n=1,2,3, \cdots$, where $f_{n}$ is a representative map for $\alpha_{n}$.

Now let $A_{i}, B_{i}(i \in J=\{1,2,3, \cdots\})$ be simply-connected spaces with base points; then

$$
X=\bigvee_{i \in J}\left(A_{i} \vee B_{i}\right)=\left(\bigvee_{i \in J} A_{i}\right) \vee\left(\bigvee_{i \in J} B_{i}\right)
$$

is also simply-connected. Let $m, n>1$ be fixed integers, and as before let $q=m+n-1$.

Theorem 2. Let $\alpha_{i} \in \pi_{m}\left(A_{i}, *\right), \beta_{i} \in \pi_{n}\left(B_{i}, *\right)$, and $\gamma=\sum_{i \in J}\left[\alpha_{i}, \beta_{i}\right]$ $\in \pi_{q}(X, *)$. Then $\omega_{Q}(\gamma) \neq 0$ if $\omega_{Q}\left(\alpha_{i}\right) \neq 0, \omega_{Q}\left(\beta_{i}\right) \neq 0$ for an infinite number of values of $i$. (We recall that $\omega_{Q}$ is the composition

$$
\left.\pi_{r}(X, *) \rightarrow H_{r}(X) \rightarrow H_{r}(X ; Q) \omega\right) .
$$

Proof. If $\omega_{Q}\left(\alpha_{i}\right) \in H_{m}\left(A_{i} ; Q\right)$ is not zero, we may choose a cohomology class $u_{i} \in H^{m}\left(A_{i} ; Q\right)$ such that $\left\langle u_{i}, \omega_{Q}\left(\alpha_{i}\right)\right\rangle \neq 0$. Similarly if $\omega_{Q}\left(\beta_{i}\right) \neq 0$ we can choose $v_{i} \in H^{n}\left(B_{i} ; Q\right)$ such that $\left\langle v_{i}, \omega_{Q}\left(\beta_{i}\right)\right\rangle \neq 0$.

First suppose that $\omega(\gamma) \in H_{q}(X)$ is zero: we derive a contradiction. By Lemma 1 there is a map $f: S^{q} \rightarrow X$ representing the homotopy class $\gamma$, and which can be factorised through a map $g$ of a finite polyhedron $K$ of dimension less than $q$ into $X$. It follows that

$$
f^{*}: B^{q}(X ; Q) \rightarrow H^{q}\left(S^{q} ; Q\right)
$$

is trivial, and since $u_{i} \cup v_{j}=0$, the functional cup-products 


$$
u_{i} \bigcup_{f} v_{j} \in H^{q}\left(S^{q} ; Q\right)
$$

are defined whenever $u_{i}, v_{j}$ are defined. It is easy to show from Lemma 2 , by making use of the projections $p_{i, j}: X \rightarrow A_{i} \vee B_{j}$, that

$$
\begin{aligned}
u_{i} \bigcup_{f} v_{j}=0 & \text { if } i \neq j, \\
\neq 00 & \text { if } i=j .
\end{aligned}
$$

We have supposed that $u_{i} \cup_{f} v_{i}$ has been defined for an infinite number of values of $i$.

Suppose $f$ is factored into $g \circ h$, where $g: K \rightarrow X$. Then, since $\operatorname{dim}(K)<q, H^{q}(K ; Q)=0$, and the functional cup-product $\cup_{h}$ defines a bilinear pairing

$$
H^{m}(K ; Q) \otimes H^{n}(K ; Q) \rightarrow H^{q}\left(S^{q} ; Q\right),
$$

such that

$$
\begin{aligned}
\left(g^{*} u_{i}\right) \bigcup_{h}\left(g^{*} v_{\jmath}\right)=u_{i} \bigcup_{f} v_{j} & =0 \quad \text { if } i \neq j, \\
& \neq 0 \quad \text { if } i=j .
\end{aligned}
$$

This implies that infinitely many of the elements $g^{*} u_{i}$ are linearly independent; this is impossible since $K$ is a finite complex. Hence $\omega(\gamma) \neq 0$.

REMARK. Thus far any field could be substituted for the rationals $Q$. The next step does not work for fields of finite characteristic.

Let $p$ be any nonzero integer. Then

$$
p \gamma=\sum_{i \in J}\left[p \alpha_{i}, \beta_{i}\right]
$$

and clearly $\omega_{Q}\left(p \alpha_{i}\right)=p \omega_{Q}\left(\alpha_{i}\right)$ is not zero if $\omega_{Q}\left(\alpha_{i}\right) \neq 0$. Therefore the argument shows that $\omega(p \gamma) \neq 0$, that is, $\omega(\gamma)$ is not of finite order. But the kernel of the coefficient homomorphism $H_{q}(X) \rightarrow H_{q}(X ; Q)$ is the torsion subgroup of $H_{q}(X)$. Therefore $\omega(\gamma)$ is not in this kernel, and $\omega_{Q}(\gamma) \neq 0$. This completes the proof.

3. Proof of Theorem 1. Let $A_{n}=B_{n}=\mathrm{V}_{i \in J} S_{i}^{r}$, and let $X=\mathrm{V}_{n \in J}\left(A_{n} \bigvee B_{n}\right)$. Then $X$ is homeomorphic to $A_{n}$; hence if the group $\omega_{Q}\left(\pi_{p}(X)\right)$ is not zero it cannot be countable, for it must contain the direct product $\prod_{n \in J} \omega_{Q}\left(\pi_{p}\left(A_{n}\right)\right)$.

We now construct, by induction on $t$, elements

$$
\gamma_{t} \in \pi_{t(r-1)+1}(X, *)
$$

such that $\omega_{Q}\left(\gamma_{t}\right) \neq 0$ in $H_{t(r-1)+1}(X ; Q)$. This is certainly possible for 
$t=1$. Therefore there are elements $\alpha_{n} \in \pi_{r}\left(A_{n}, *\right)$ with $\omega_{Q}\left(\alpha_{n}\right) \neq 0$. Suppose that $\boldsymbol{\gamma}_{1}, \cdots, \gamma_{t-1}$ have been constructed. Corresponding to $\gamma_{t-1}$ there are elements

$$
\beta_{n, t-1} \in \pi_{(t-1)(r-1)+1}\left(B_{n}, *\right)
$$

with $\omega_{Q}\left(\beta_{n, t-1}\right) \neq 0$. According to Theorem 2 the element

$$
\gamma_{t}=\sum_{n \in J}\left[\alpha_{n}, \beta_{n, t-1}\right] \in \pi_{t(r-1)+1}(X, *)
$$

satisfies $\omega_{Q}\left(\gamma_{t}\right) \neq 0$. This completes the induction and the proof of Theorem 1.

By a similar means we may prove

TheOREM 3. Let $A_{n}(n \in J)$ be simply-connected spaces such that for some $r>1$

$$
0 \neq \omega_{Q}\left(\pi_{r}\left(A_{n}, *\right)\right) \subset H_{r}\left(A_{n} ; Q\right) .
$$

Then $H_{q}\left(\mathrm{~V}_{n \in J} A_{n}, Q\right)$ is not countable for $q \equiv 1 \bmod (r-1), q>1$.

\section{REFERENCES}

1. S. Eilenberg, On the problems of topology, Ann. of Math. 50 (1949), 247-260.

2. N. E. Steenrod, Cohomology invariants of mappings, Ann. of Math. 50 (1949), 954-988.

3. J. H. C. Whitehead, $A$ certain exact sequence, Ann. of Math. 52 (1951), 51-110.

MaNChester UNIVERSITY AND

Princeton University 